\title{
Glomus Tumor: An Anomalous Cause of Pain in the Finger
}

\author{
1 Jayakrishnan K Narayana Kurup, ${ }^{2}$ Ashwath M Acharya, ${ }^{3}$ Anil K Bhat
}

\begin{abstract}
Glomus tumor constitutes 1 to $5 \%$ of all hand tumors. They present with the classical triad of severe pain, point tenderness, and cold sensitivity. The diagnosis is mainly clinical, but very often it is missed because of lack of knowledge or inadequate clinical examination. We present the case of a 43-year-old woman with glomus tumor of the middle finger with a short discussion on the diagnosis with clinical images of the tumor. Glomus tumor should be in the differential diagnosis in patients presenting with pain in the finger.
\end{abstract}

Keywords: Benign, Cold insensitivity, Glomus tumor.

How to cite this article: Narayana Kurup JK, Acharya AM, Bhat AK. Glomus Tumor: An Anomalous Cause of Pain in the Finger. Int J Recent Surg Med Sci 2016;2(2):106-107.

Source of support: Nil

Conflict of interest: None

\section{CASE REPORT}

A 43-year-old housewife came to the us with diffuse pain in the middle finger of right hand since 5 months. The pain was more during night and was relieved on taking nonsteroidal anti-inflammatory drugs. She had consulted two general practitioners before coming to us and was treated symptomatically with medications since no abnormality was detected during examination and X-rays. With detailed clinical examination, the patient's pain was localized to the nail bed area (Fig. 1). On pressing with a pin head, patient had excruciating pain localized to the nail bed (Love's test positive). There was no change in the pain perception on release of the tourniquet applied to the elevated arm (Hildreth's test negative). Radiographs of the middle finger were normal. A magnetic resonance imaging (MRI) scan was done to confirm the clinical diagnosis of glomus tumor. Magnetic resonance imaging showed a well-defined oval enhancing lesion measuring $7 \times 5 \times 5 \mathrm{~mm}$ within the soft tissue of the dorsal and ulnar aspect of the terminal phalanx of the middle finger (subungual region). The lesion was isointense to muscles on $\mathrm{T} 1$ and hyperintense on T2 and Proton density fat

\footnotetext{
${ }^{1}$ Senior Resident, ${ }^{2}$ Associate Professor, ${ }^{3}$ Professor

${ }^{1-3}$ Department of Orthopaedics, Kasturba Medical College Manipal University, Manipal, Karnataka, India

Corresponding Author: Jayakrishnan K Narayana Kurup Senior Resident, Department of Orthopaedics, Kasturba Medical College, Manipal University, Manipal, Karnataka, India, Phone: +919449689558, e-mail: jkdoctorjk@gmail.com
}

suppressed (PDFS) sequences, causing scalloping and thinning of the underlying cortex of the terminal phalanx. Patient underwent complete excision of the tumor. Transungal approach was used with removal of nail plate and a portion of the nail bed. A well-circumscribed reddish mass with a bluish hue was excised (Figs 2 and 3). Nail bed was repaired by using a toe nail bed graft from the big toe (Figs 4 and 5). Patient had complete relief of pain after the excision surgery.

\section{DISCUSSION}

Glomus body is a perivascular structure formed by arteriovenous anastomosis that arises from the neuromyoarterial tissue located mainly in the subungal area of finger

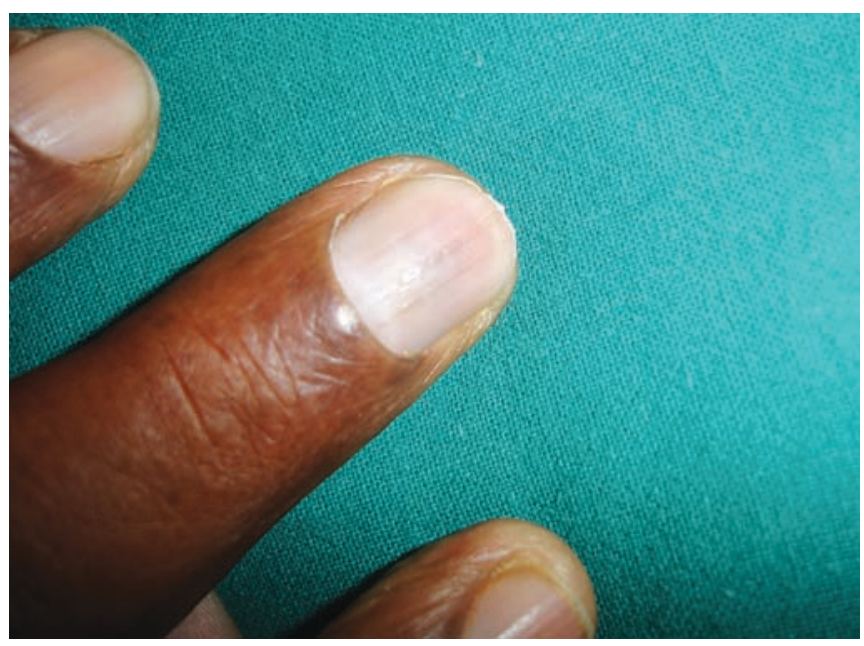

Fig. 1: Right middle finger tip with a bluish hue below the nail plate

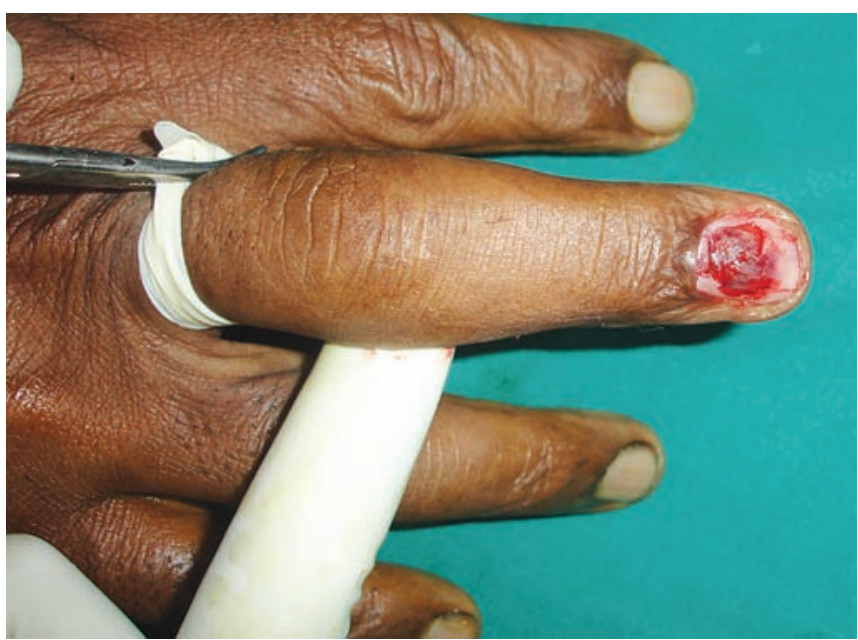

Fig. 2: Nail plate and nail bed removed exposing the tumor mass 


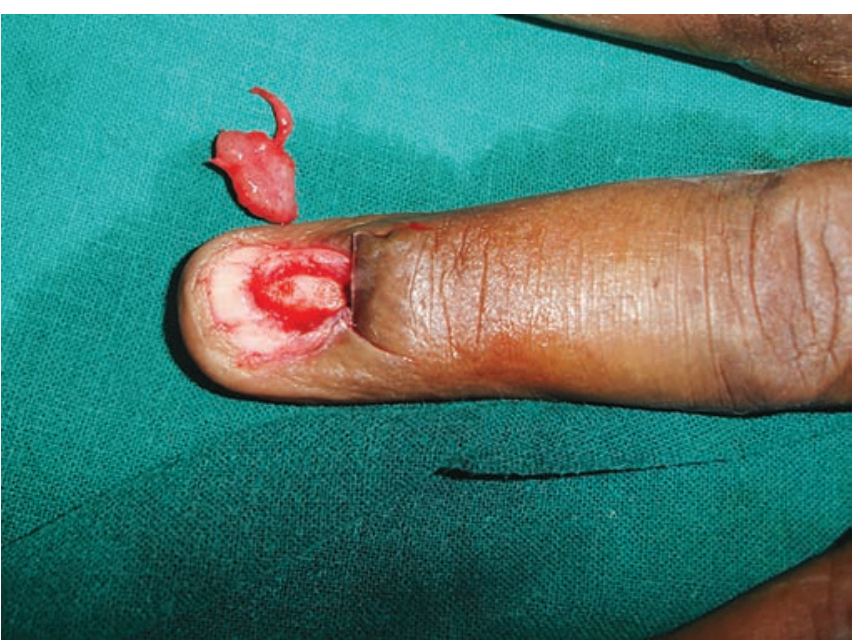

Fig. 3: Excision of the complete tumor mass with defect in the nail bed

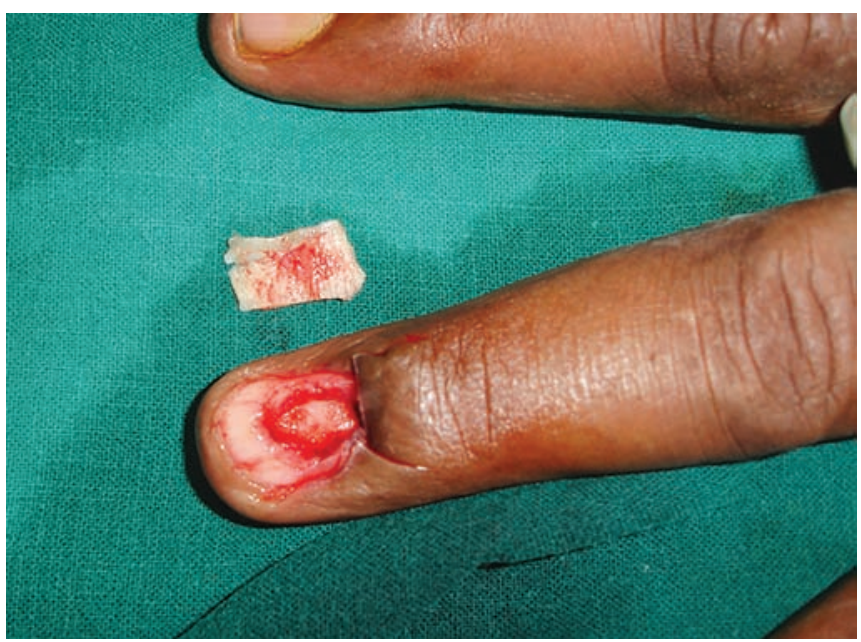

Fig. 5: Harvested nail bed graft prepared for the defect in the nail bed of the middle finger

tips. The main function of glomus body is temperature regulation of fingers. Glomus tumors are benign hamartomas arising from the glomus body. Glomus tumor constitutes 1 to $5 \%$ of all hand tumors. ${ }^{1}$ Glomus tumors are seen in middle-aged women between 30 and 50 years of age. ${ }^{2}$ They are usually solitary tumors presenting with a classical triad of severe pain, point tenderness, and cold sensitivity. ${ }^{3}$ Other features commonly seen are nail deformity, bluish discoloration, and a palpable nodule. Diagnosis of glomus tumor is mainly clinical. Love's test, Hildreth's test, and cold sensitivity are the clinical tests described for diagnosing glomus tumor. In a positive Love's test, the patient experiences excruciating pain when the tumor is pressed with a pin head and the pain is relieved on releasing the pressure from the tumor. Cold sensitivity test is positive when the patient experiences severe pain around the tumor on immersing the hand in cold water. In Hildreth's test, the patient's

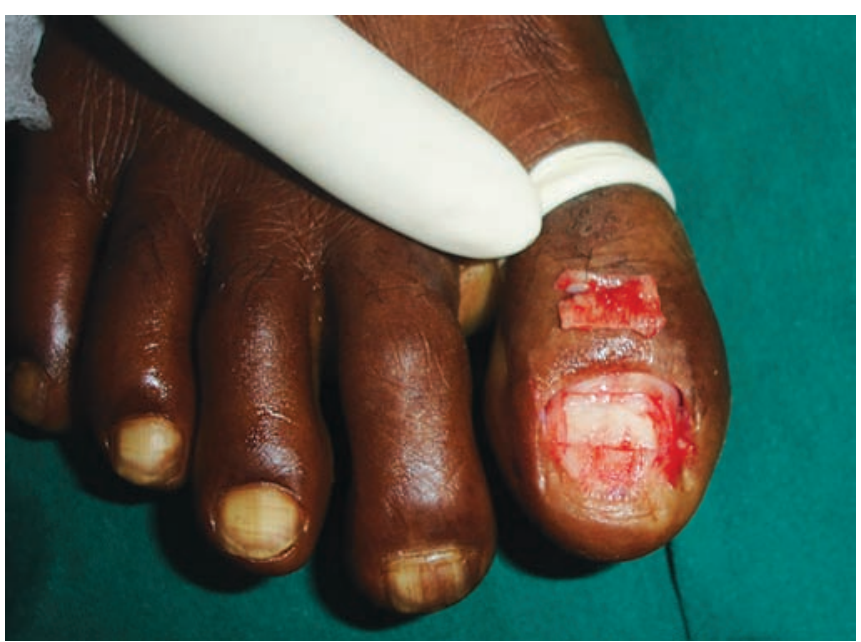

Fig. 4: Harvesting of nail bed graft from the big toe

hand is elevated for some time for exsanguination and a sphygmomanometer cuff/tourniquet is inflated in the arm. In a positive test, the patient feels reduction in the pain and tenderness after inflating the tourniquet cuff. Radiographs and MRI are routinely used for diagnosis and localization of glomus tumor. Excision of the tumor is the treatment of choice for glomus tumor. Surgical incisions and scar retractions can result in significant nail deformity. We have used a nail bed graft from the toe to minimize the nail deformity. ${ }^{4}$ Recurrences are not common unless the excision of tumor was not complete or there were multiple tumors which were missed during initial surgery. Glomangiosarcoma is a very rare slowgrowing malignant variant of the glomus tumor causing local recurrence with less chance of metastasis.

\section{CONCLUSION}

Glomus tumor presents with a classical triad of severe pain, point tenderness, and cold sensitivity. Diagnosis of glomus tumor is mainly clinical. Glomus tumor should be in the differential diagnosis in patients presenting with pain in the finger. Excision of the tumor is the treatment of choice.

\section{REFERENCES}

1. Van ruyssevelt CE, Vranckx P. Subungual glomus tumor: emphasis on MR angiography. AJR Am J Roentgenol 2004 Jan;182(1):263-264.

2. Carroll RE, Berman AT. Glomus tumors of the hand. J Bone Joint Surg 1972 Jun;54A(4):691-703.

3. Bhaskaranand K, Navadgi BC. Glomus tumour of the hand. J Hand Surg Br 2002 Jun;27(3):229-231.

4. Pessa JE, Tsai TM, Li Y, Kleinert HE. The repair of nail deformities with nonvascularized nail bed grafts: indications and results. J Hand Surg Am 1990 May;15A(3):466-470. 